\title{
Specific strains of Streptococcus mutans, a pathogen of dental caries, in the tonsils, are associated with IgA nephropathy
}

\author{
Seigo Ito $^{1 *}$, Taro Misaki ${ }^{2,3}$, Shuhei Naka ${ }^{4}$, Kaoruko Wato ${ }^{5}$, Yasuyuki Nagasawa ${ }^{6}$, \\ Ryota Nomura ${ }^{5}$, Masatoshi Otsugu ${ }^{5}$, Michiyo Matsumoto-Nakano ${ }^{4}$, Kazuhiko Nakano $\mathbb{D}^{5}$, \\ Hiroo Kumagai ${ }^{1} \&$ Naoki Oshima $^{1}$
}

Streptococcus mutans is known to be a major causative agent of dental caries, and strains expressing the cell surface collagen-binding $\mathrm{Cnm}$ protein contribute to the development of several systemic diseases. A relationship between tonsillar immunity and glomerulonephritis has been recognized in IgA nephropathy (IgAN), and specific pathogens may have effects on tonsillar immunity (mucosal immunity). Here, we present findings showing a relationship between the presence of $C$ nm-positive $S$. mutans strains in the tonsils of IgAN patients and IgAN condition/pathogenesis. Analyses of tonsillar specimens obtained from patients with IgAN $(n=61)$ and chronic tonsillitis (controls; $n=40)$ showed that the $\mathrm{Cnm}$ protein-positive rate was significantly higher in IgAN patients. Among IgAN patients, the tonsillar $\mathrm{Cnm}$-positive group ( $\mathrm{n}=15$ ) had a significantly higher proportion of patients with high urinary protein $(>1.5 \mathrm{~g} / \mathrm{gCr}$ ) and lower serum albumin level than the $\mathrm{Cnm}$-negative group ( $\mathrm{n}=46$ ). Additionally, Cnm protein and CD68, a common human macrophage marker, were shown to be merged in the tonsils of IgAN patients. These findings suggest that $\mathrm{Cnm}$-positive $\mathrm{S}$. mutans strains in the tonsils may be associated with severe IgAN.

Patients with Immunoglobulin A nephropathy (IgAN), the most common cause of primary glomerulonephritis throughout the world ${ }^{1,2}$, have a poor renal prognosis ${ }^{3}$. Without undergoing specific therapy, more than one-third of these patients progress to end-stage kidney disease within 20 years ${ }^{4,5}$. Unfortunately, few disease-targeted treatment options are available because the pathogenesis of IgAN remains to be fully clarified ${ }^{6}$.

IgAN patients are often presented with macroscopic hematuria with upper respiratory infections, such as tonsillitis ${ }^{7}$. Hotta et al. were the first to proposed that a tonsillectomy along with steroid pulse therapy for IgAN patients would not only decrease hematuria/proteinuria but also result in improved remission rates ${ }^{8}$. The results of a recently published large-scale clinical study indicated that tonsillectomy is effective as treatment for IgAN ${ }^{9}$. Furthermore, clinical observations have indicated a relationship between tonsillar immunity and pathogenesis ${ }^{10-12}$. Tonsillar immunity is considered mucosal immunity: when exposed to pathogens (e.g. bacteria and their constituents), antigen-presenting cells such as macrophages become the starting point and cause B-cells and plasma cells to produce antibodies including IgA.

In comparisons of tonsils obtained from IgAN patients with those from tonsillar hyperplasia cases, the relative abundance of specific bacteria was found to be significantly different ${ }^{13}$, suggesting the tonsillar immune system or the hosts' genetic make-up, other epigenetics or environmental factors behind the colonization of specific bacteria in the tonsils are important with respect to the pathogenesis of IgAN. Indeed, some bacteria, specifically

\footnotetext{
${ }^{1}$ Department of Nephrology and Endocrinology, National Defense Medical College, Tokorozawa, Saitama, Japan. ${ }^{2}$ Division of Nephrology, Seirei Hamamatsu General Hospital, Hamamatsu, Shizuoka, Japan. ${ }^{3}$ Department of Nursing, Faculty of Nursing, Seirei Christopher University, Hamamatsu, Shizuoka, Japan. ${ }^{4}$ Department of Pediatric Dentistry, Okayama University Graduate School of Medicine, Dentistry and Pharmaceutical Sciences, Okayama, Okayama, Japan. ${ }^{5}$ Department of Pediatric Dentistry, Division of Oral infection and Disease Control, Osaka University Graduate School of Dentistry, Suita, Osaka, Japan. ${ }^{6}$ Division of Kidney and Dialysis, Department of Internal Medicine, Hyogo College of Medicine, Nishinomiya, Hyogo, Japan. *email: sitoh@ndmc.ac.jp
} 


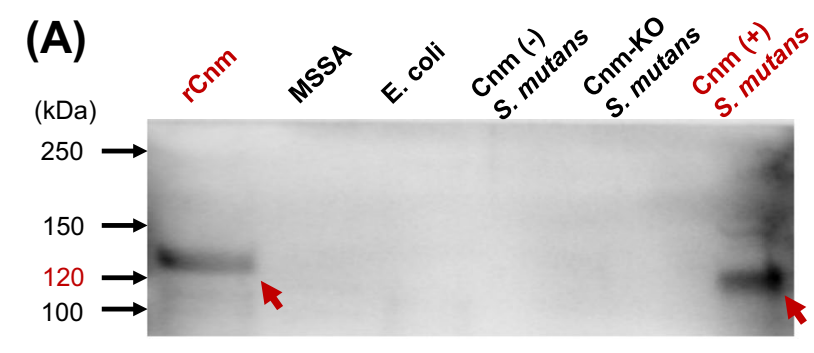

(B)

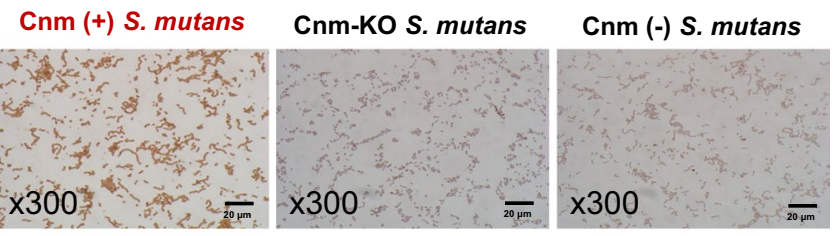

\section{(C)}

rCnm protein unsaturated

\section{0x rCnm protein saturated}

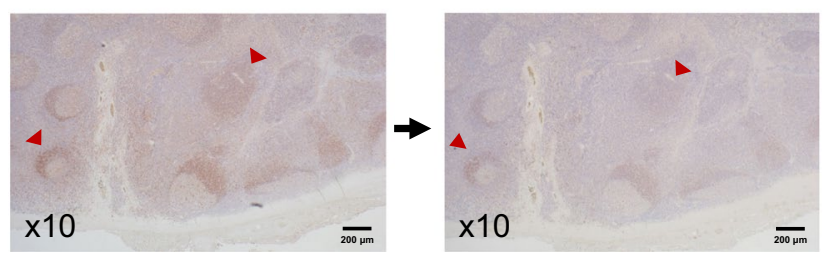

Figure 1. Construction of Cnm antiserum. (A) Western blotting of recombinant $\mathrm{Cnm}(\mathrm{rCnm})$ protein and cellsurface proteins of Cnm-negative strains: methicillin-susceptible Staphylococcus aureus (MSSA), Escherichia coli, $\mathrm{Cnm}(-)$ S. mutans, and Cnm-KO S. mutans, and the Cnm-positive strain Cnm (+) S. mutans. Arrows indicate positive bands at $120 \mathrm{kDa}$. (B) Culture smears with $\mathrm{Cnm}(+)$ S. mutans, Cnm-KO S. mutans, and Cnm (-) S. mutans. Immunohistochemistry findings showed that the $\mathrm{Cnm}$-positive strain was stained and the $\mathrm{Cnm}$-negative strains were not. (C) Serial sections of tonsils obtained from IgAN patient. Immunoperoxidase staining for Cnm, using $\mathrm{Cnm}$ antiserum unsaturated or saturated with $\mathrm{rCnm}$ protein, showed that saturating with $\mathrm{rCnm}$ protein diminished staining with $\mathrm{Cnm}$ antiserum (arrowheads).

periodontitis-related bacteria, have been referred to as the causal antigens or exacerbation factors in the pathogenesis of the disease $\mathrm{e}^{14-18}$.

Streptococcus mutans, categorized as a Gram-positive facultative anaerobic oral streptococcal species, has been recognized as a major pathogenic agent in the development of dental caries in humans ${ }^{19}$. Furthermore, $S$. mutans has been reported to trigger infective endocarditis (IE) and has occasionally been isolated from blood specimens obtained from IE patients ${ }^{20}$. Some $S$. mutans strains express the cell surface collagen-binding protein $\mathrm{Cnm}$, which is encoded by the $\mathrm{cnm}$ gene. Cnm shows an extracellular matrix-binding capability and can be a pathogenetic factor related to $\mathrm{IE}^{21}$. Previously, we reported that $S$. mutans strains showing Cnm expression were associated with aggravation of various systemic diseases, such as cerebral hemorrhage ${ }^{22-24}$, non-alcoholic steatohepatitis $^{25}$, and inflammatory bowel disease ${ }^{19}$. Moreover, our additional studies showed a significantly higher carrier rate of Cnm-positive $S$. mutans strains in the saliva of IgAN patients than in that of non-IgAN controls, and Cnm-positive S. mutans strains present in the oral cavity may have effects on the severity of $\operatorname{IgAN}{ }^{26-28}$.

The purpose of this study was to confirm the presence of Cnm-positive S. mutans in the tonsils, which was suggested to be more related to the severity of IgAN, and to investigate the correlation with clinical background.

\section{Results}

Generation of $\mathrm{Cnm}$ antiserum. To confirm the immunoreactive specificity of obtained antisera to Cnmpositive S. mutans, we used Cnm (+) S. mutans, Cnm (-) S. mutans, and Cnm-KO S. mutans cells. Methicillinsusceptible Staphylococcus aureus (MSSA) and Escherichia coli cells were used as negative controls. Western blot analysis demonstrated a positive band in $\mathrm{Cnm}(+) S$. mutans whole cell extracts, whereas no bands were found in the MSSA, E. coli, Cnm (-) S. mutans, or Cnm-KO S. mutans extracts (Fig. 1A).

Immunohistochemistry findings of a cultured bacterial smears for $\mathrm{Cnm}$ showed a positive reaction for $\mathrm{Cnm}$ (+) S. mutans and a negative reaction for Cnm-KO S. mutans and Cnm (-) S. mutans (Fig. 1B). To confirm a specific reaction of $\mathrm{Cnm}$ antiserum to $\mathrm{Cnm}$-positive $S$. mutans in the tonsil tissues, immunoperoxidase staining for $\mathrm{Cnm}$ was performed with serial tonsillar sections using $\mathrm{Cnm}$ antiserum either unsaturated or saturated with recombinant $\mathrm{Cnm}(\mathrm{rCnm})$ protein, which showed that saturation with $\mathrm{rCnm}$ protein diminished tonsillar staining by $\mathrm{Cnm}$ antiserum (Fig. 1C). 
(A)

(i) Positive Case

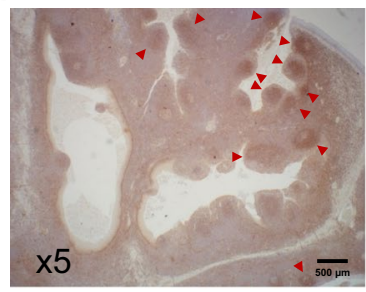

(ii) Negative Case

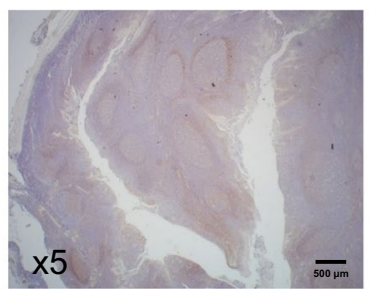

Threshold drawing

(B) Tonsils IHC image

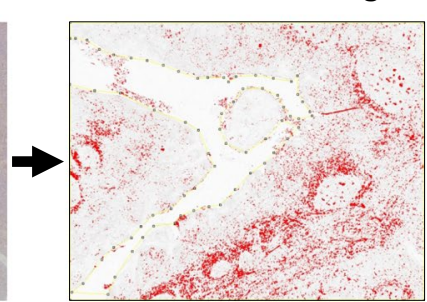

(C)

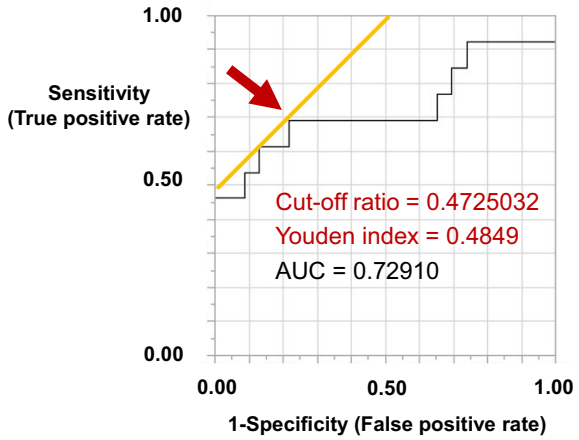

Figure 2. Assessment of degrees of Cnm-positive S. mutans in the tonsils from patients with IgAN and chronic tonsillitis. (A) Tonsillar sections were obtained from patients with IgAN and chronic tonsillitis. The degree of tonsillar immunoperoxidase staining for Cnm varied among the patients and disease group. (i) Representative tonsil from an IgAN patient (positive Case) showing strong staining, including germinal centers (arrowheads). (ii) Representative tonsil from an IgAN patient (negative Case) showing weak staining, even in the germinal centers. (B) Measurement of ratio of Cnm-positive area to the entire tonsil. Images taken at low magnification were converted to 8-bit red, green, and blue (RGB) images, and a threshold drawing was then generated using the image processing program, Fiji/ImageJ. (C) Receiver operating characteristic (ROC) curves for the ratio of $\mathrm{Cnm}$-positive area to the entire tonsil for predicting $\mathrm{cnm}$ positivity in salivary specimens from IgAN patients by PCR. Arrow indicates the cut-off ratio yielding maximum sensitivity and specificity for the prediction of Cnm positivity in the specimen. The optimal cut-off ratio was $0.472(\mathrm{n}=36$, area under the curve $=0.72910$; sensitivity $=61.5 \%$; specificity $=87.0 \%$ ).

Assessment of the degree of $\mathrm{Cnm}$ positivity in the tonsils from IgAN and chronic tonsillitis patients. The degree of immunoperoxidase staining for $\mathrm{Cnm}$ in tonsillar sections varied by patient and disease group (Fig. 2A). Figure 2A shows demonstrative findings from a strongly positive (i) and weakly negative case (ii). As for the positive case, the entire area including the germinal centers was well stained and the germinal centers were not stained, whereas, in the negative case, the mantle zone around them was only weakly stained. To evaluate the ratio of Cnm-positive area to the entire tonsil, images obtained with low magnification were converted to 8-bit red, green, and blue images, from which a threshold drawing was generated (Fig. 2B).

A receiver operating characteristic curve for identifying the cut-off ratio (Cnm-positive area to the entire tonsil) that provides optimal separation between the tonsillar $\mathrm{Cnm}$-positive and $\mathrm{Cnm}$-negative groups is shown in Fig. 2C. The optimal cut-off ratio for yielding maximum sensitivity and specificity for the prediction of $\mathrm{cnm}$ positivity in the saliva was $0.472(n=36$, area under the curve $=0.72910$; sensitivity $61.5 \%$, specificity $87.0 \%)$.

Characteristics of IgAN and chronic tonsillitis (control) groups. The interval between the kidney biopsy and tonsillectomy procedures was $11.2 \pm 18.8$ months (mean \pm SD). The age of the IgAN group was significantly higher than that of the control group $(p<0.05)$. Additionally, the percentages of urinary protein excretion and urinary occult blood were significantly higher in the IgAN group $(p<0.0001)$. The estimated glomerular filtration rate (eGFR) tended to be lower in the IgAN than in the control group, although the difference was not 


\begin{tabular}{|c|c|c|c|}
\hline Years & $\begin{array}{l}\text { IgAN group } \\
(\mathrm{n}=61)\end{array}$ & $\begin{array}{l}\text { Control group } \\
(\mathrm{n}=40)\end{array}$ & $p$-value \\
\hline Age (year; mean $\pm \mathrm{SD}$ ) & $35.9(32.9-38.9)$ & $30.3(27.4-33.2)$ & 0.0359 \\
\hline $\operatorname{Sex}(M / F)$ & $31 / 30$ & $28 / 12$ & 0.0655 \\
\hline Cnm-positive area/total tonsillar area ratio $($ mean $\pm S D)$ & $0.32 \pm 0.17$ & $0.04 \pm 0.06$ & $<0.0001$ \\
\hline Positive rate of Cnm-positive S. mutans in tonsils (\%) & 24.5 & 0.0 & 0.0003 \\
\hline Serum creatinine $(\mathrm{mg} / \mathrm{dL} ;$ mean $\pm \mathrm{SD})$ & $0.8 \pm 0.2$ & $0.7 \pm 0.1$ & 0.4764 \\
\hline eGFR $(\mathrm{mL} / \mathrm{min} ;$ mean $\pm \mathrm{SD})$ & $83.4 \pm 19.5$ & $90.9 \pm 12.9$ & 0.0658 \\
\hline$\%$ of urinary protein $1+$ or higher & 78.6 & 2.5 & $<0.0001$ \\
\hline$\%$ of urinary occult blood $1+$ or higher & 95.0 & 4.7 & $<0.0001$ \\
\hline
\end{tabular}

Table 1. Characteristics of IgAN and chronic tonsillitis (control) groups. SD: standard deviation, eGFR: estimated glomerular filtration rate Bold values indicate statistical significance at $p<0.05$.

significant (Table 1). The Cnm-positive area/total tonsillar area ratio was significantly higher in the IgAN group than the control group $(p<0.0001)$, as was the positive rate for $\mathrm{Cnm}$ (Cnm-positive patients/total patients) in the tonsils $(24.5 \%$ vs. $0.0 \%, p=0.0003)$ (Table 1$)$.

Comparison between IgAN patients in $\mathrm{Cnm}$-positive and $\mathrm{Cnm}$-negative groups. For this comparison, we analyzed 61 IgAN patients (mean age, $35.9 \pm 11.7$ years; 31 males, 30 females). The percentage of those with urinary protein at $1.5 \mathrm{~g} / \mathrm{gCr}$ or greater was higher in the $\mathrm{Cnm}$-positive group (26.6\% vs. $4.3 \%$, $p=0.0280)$, whereas serum albumin in that group was significantly lower $(p=0.0256)$ (Table 2$)$.

There were no significant differences in age, sex, duration from onset to treatment (only tonsillectomy or combination of tonsillectomy and systemic steroid administration for approximately 1 year), body mass index, blood pressure (systolic, diastolic), serum total cholesterol, serum low-density lipoprotein cholesterol, serum triglyceride, serum creatinine, eGFR, serum IgA, and urinary red blood cell (RBC) score between the groups (Table 2).

Analysis of kidney histology in IgAN patients. A total of 44 kidney biopsy samples from 61 IgAN patients (Cnm-positive, $\mathrm{n}=10$; Cnm-negative, $\mathrm{n}=34$ ) were analyzed, and histological findings were compared based on the Oxford classification. The segmental glomerulosclerosis and tubular atrophy/interstitial fibrosis scores in the Cnm-positive group tended to be higher (Table 3).

Cnm antiserum staining of glomeruli in IgAN patients. Cnm immunostaining of the kidney specimens was conducted on 119 cases, including 57 cases of IgAN patients in whom both tonsillar and kidney specimens were available, and 62 cases of IgAN patients, in whom only kidney specimens were available. Most of these IgAN patients did not show positive glomerular staining for $\mathrm{Cnm}$, with only 4 suspected to have positive staining. However, we concluded that those were false positive findings.

Relationship between galactose-deficient IgA1 glomerular deposition and tonsillar Cnm-positive rate in IgAN patients. Immunofluorescent (IF) staining for galactose-deficient IgA1 (Gd-IgA1) showed varying intensity (Fig. 3A). The rate of glomerular Gd-IgA $1+$ was $84.6 \%$ in tonsils from the Cnm-positive group and $53.6 \%$ in those from the Cnm-negative group ( $p=0.0567)$ (Fig. 3B).

Double IF staining for $\mathrm{Cnm}$ and CD68 in tonsillar sections from IgAN patients. Double IF staining for Cnm (Alexa Fluor 488: green) and CD68, a macrophage marker (Alexa Fluor 594: red), was performed using tonsillar sections that had been stained with immunoperoxidase for $\mathrm{Cnm}$. Cnm staining was observed mainly in the germinal center or the surrounding area. Figure 4A shows a representative Cnm-positive germinal center (yellow circle) at a high magnification. CD68 staining of the same area is shown in Fig. 4B, whereas DAPI is shown in Fig. 4C, with a merged image presented in Fig. 4D. Arrows indicate the colocalization of Cnm with CD68.

\section{Discussion}

This is the first study to indicate that Cnm-positive $S$. mutans strains in the tonsils may be associated with severe IgAN. Our findings showed that the Cnm-positive area/total tonsillar area ratio was significantly higher in IgAN patients than in the control group (Table 1). Furthermore, Cnm protein was observed in the tonsils (Fig. 2A) but not in the kidneys of IgAN patients, whereas $\mathrm{Cnm}$ expression in the tonsils was associated with urinary protein level (Table 2). The degree of positivity for Gd-IgA1 in the kidneys tended to be higher in Cnm-positive patients than in Cnm-negative patients. Moreover, our results based on IF staining of the tonsils indicate the possibility of macrophage coexistence with Cnm-positive $S$. mutans. According to earlier reports, Cnm-positive rates in the saliva and areas affected by dental caries had significant correlations with urinary protein level in IgAN patients ${ }^{26,27}$. Nevertheless, those studies did not reveal a possible mechanism related to the involvement of Cnm-positive $S$. mutans in the development and severity of IgAN. Our findings suggest the association of Cnm-positive $S$. mutans with the severity of IgAN via their colonization in the tonsils.

The Cnm-positive group had a significantly higher proportion of patients with high urinary protein level and lower serum albumin level than the Cnm-negative group, while both tended to have a lower eGFR (Table 2). Proteinuria causes hypoalbuminemia, and both may sometimes coincide with the presence of IgAN ${ }^{29}$. Generally, urinary protein load in the renal tubules causes a decrease in eGFR via a decrease in post-glomerular flow ${ }^{30}$. 


\begin{tabular}{|c|c|c|c|}
\hline Characteristics & $\begin{array}{l}\text { Cnm-positive } \\
(\mathrm{n}=15)\end{array}$ & $\begin{array}{l}\text { Cnm-negative } \\
(n=46)\end{array}$ & p-value \\
\hline Age (year; mean $\pm \mathrm{SD}$ ) & $39.0(32.1-45.9)$ & $34.9(31.5-38.3)$ & 0.2650 \\
\hline $\operatorname{Sex}(M / F)$ & $9 / 6$ & $22 / 24$ & 0.5541 \\
\hline Duration from onset to treatment (year; mean $\pm S D$ ) & $5.2 \pm 5.4$ & $7.5 \pm 9.4$ & 0.9112 \\
\hline $\mathrm{BMI}\left(\mathrm{kg} / \mathrm{m}^{2} ;\right.$ mean $\left.\pm \mathrm{SD}\right)$ & $23.5 \pm 5.9$ & $21.3 \pm 2.7$ & 0.4754 \\
\hline Systolic blood pressure (mmHg; mean $\pm \mathrm{SD}$ ) & $116.6 \pm 17.1$ & $117.8 \pm 17.7$ & 0.6817 \\
\hline Diastolic blood pressure $(\mathrm{mmHg} ;$ mean $\pm \mathrm{SD})$ & $70.0 \pm 12.5$ & $70.8 \pm 13.4$ & 0.7779 \\
\hline Serum albumin (g/dL; mean $\pm \mathrm{SD})$ & $3.8 \pm 0.4$ & $4.1 \pm 0.3$ & 0.0256 \\
\hline Serum total cholesterol (mg/dL; mean $\pm \mathrm{SD})$ & $216.7 \pm 82.2$ & $193.3 \pm 28.9$ & 0.5082 \\
\hline Serum LDL cholesterol (mg/dL; mean \pm SD) & $140.1 \pm 83.7$ & $111.9 \pm 22.7$ & 0.3461 \\
\hline Serum triglyceride $(\mathrm{mg} / \mathrm{dL} ;$ mean $\pm \mathrm{SD})$ & $179.2 \pm 167.7$ & $104.8 \pm 58.3$ & 0.0789 \\
\hline Serum creatinine $(\mathrm{mg} / \mathrm{dL} ;$ mean $\pm \mathrm{SD})$ & $0.8 \pm 0.2$ & $0.7 \pm 0.1$ & 0.1472 \\
\hline eGFR $\left(\mathrm{mL} / \mathrm{min} / 1.73 \mathrm{~m}^{2} ;\right.$ mean $\left.\pm \mathrm{SD}\right)$ & $75.5 \pm 18.0$ & $85.4 \pm 19.5$ & 0.1067 \\
\hline Serum IgA $(\mathrm{mg} / \mathrm{dL} ;$ mean $\pm \mathrm{SD})$ & $350.1 \pm 123.6$ & $338.0 \pm 117.9$ & 0.4460 \\
\hline$\%$ Urinary protein $1.5 \mathrm{~g} / \mathrm{gCr}$ or higher & 26.6 & 4.3 & 0.0280 \\
\hline Urinary RBC score $(0 \sim 3$; mean \pm SD) & $2.1 \pm 0.7$ & $1.9 \pm 0.8$ & 0.4649 \\
\hline
\end{tabular}

Table 2. Comparison between the Cnm-positive and Cnm-negative IgAN patients. BMI: body mass index, eGFR: estimated glomerular filtration rate. SD: standard deviation, RBC: red blood cell, LDL: low-density lipoprotein Bold values indicate statistical significance at $p<0.05$.

\begin{tabular}{|l|l|l|l|}
\hline & $\begin{array}{l}\text { Cnm-positive } \\
(\mathbf{n}=\mathbf{1 0})\end{array}$ & $\begin{array}{l}\text { Cnm-negative } \\
(\mathbf{n = 3 4 )}\end{array}$ & $\boldsymbol{p}$-value \\
\hline Mesangial hypercellularity score & 0.40 & 0.38 & 0.9207 \\
\hline Segmental glomerulosclerosis score & 0.80 & 0.58 & 0.2264 \\
\hline Endocapillary hypercellularity score & 0.50 & 0.38 & 0.5108 \\
\hline Tubular atrophy/interstitial fibrosis score & 0.60 & 0.38 & 0.2272 \\
\hline
\end{tabular}

Table 3. Analysis of the kidney biopsy specimens of IgA nephropathy patients using the Oxford classification.

In IgAN patients, the predominant IgA subset in immune complexes deposited in the glomeruli was found to be IgA1, in which the O-glycan chains of the hinge region are underglycosylated (galactose-deficient IgA1) ${ }^{31,32}$. In IgAN patients, the most common extrarenal clinical manifestation is pharyngotonsillitis, which is strongly associated with exacerbation of hematuria and proteinuria ${ }^{33,34}$, phenomena that have been shown to be induced by the stimulation of the Gd-IgA1 production by infectious antigens in the tonsils ${ }^{35}$.

A rate of $1+$ or higher for glomerular Gd-IgA1 tended to be more common in the present Cnm-positive group (Fig. 3B). Furthermore, double staining of Cnm and CD68 suggested that $\mathrm{Cnm}$ may coexist with macrophages in the tonsils (Fig. 4A-D). This seems to indicate the following two possibilities in relation to Cnm-positive $S$. mutans and tonsillar macrophages. First, tonsillar macrophage phagocytose Cnm-positive $S$. mutans. During the process of tonsillar immune response against foreign antigens, macrophages engulf the antigen and subsequently process and present it to CD4 + T-cells, which are then activated. Activation of CD4 + T-cells leads to the differentiation of antigen-specific B-cells, which finally differentiate into plasma cells and secrete Gd-IgA1 ${ }^{36,37}$. Second, Cnm-positive $S$. mutans binds to the Fc $\gamma$ receptors on macrophages by forming a complex with soluble CD89. It is known that CD89, an IgA receptor, is involved in the pathology of IgAN. The mechanism is that soluble CD89 forms a complex with Gd-IgA1 and binds to the Fc receptor $\gamma$-chain on immune cells such as macrophages to initiate inflammatory signaling ${ }^{38}$. Furthermore, Streptococcus can directly bind to CD $89^{39}$. We have found the possibility of macrophage coexistence with $\mathrm{Cnm}$ in the tonsils. Few $\mathrm{Cnm}$ positive cells were detected in kidney biopsy tissues from our IgAN patients. Therefore, we suspect that the Cnm protein itself does not have direct effects on the kidneys.

This study has some limitations. First, in some of our IgAN patients, findings noting Cnm-positive S. mutans in tonsil examinations were not fully matched with the results of salivary testing. We employed immunohistochemical staining of the available paraffin-embedded tonsillar sections as the means for detecting the presence of Cnm in the tonsils. To determine the staining cut-off, we examined the correlation between Cnm positivity in the saliva and tonsils in 36 patients who had both tonsillar and salivary samples available because $\mathrm{cnm}$ detection by salivary PCR has already been established ${ }^{40}$. We found that the saliva was more likely to be positive for Cnm than the tonsils. We believe that there are several possible explanations for this result. The tonsils and saliva are in contact and the tonsils are in an anaerobic environment, whereas the saliva is in an aerobic one. S. mutans organisms prefer an aerobic environment. Additionally, we could not perform immunostaining for Gd-IgA1 in kidney sections from some IgAN patients because they had previously undergone a kidney biopsy, resulting in diagnosis at another hospital, and were subsequently referred to us for IgAN treatment, including tonsillectomy. Finally, it is possible that intraoral bacteria other than Cnm-positive S. mutans may be involved in the pathogenesis of 
(A) (i) Gd-IgA: 1+ (ii) Gd-lgA: \pm
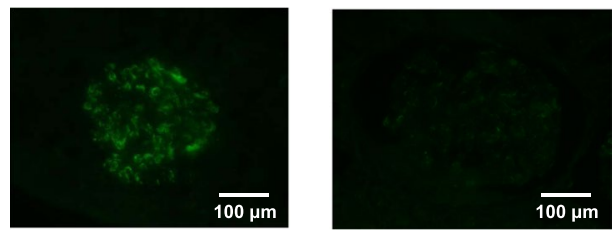

(B)

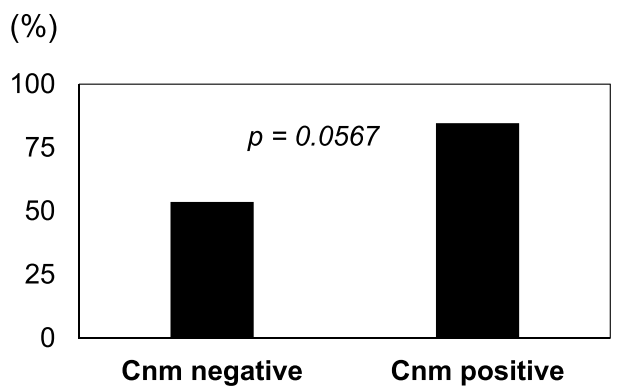

Figure 3. Relationship between glomerular deposition of galactose-deficient IgA1 (Gd-IgA1) and tonsillar Cnm-positive rate in IgAN patients. (A) IF staining for Gd-IgA1 in kidney sections from IgAN patients. Based on the degree of staining, IgAN patients were divided into two groups: (i) strong $(1+)$ and (ii) weak $( \pm)$. (B) The rate of glomerular Gd-IgA $1+$ was $84.6 \%$ in the Cnm-positive group and $53.6 \%$ in Cnm-negative group $(p=0.0567)$.

IgAN. Indeed, we previously reported that superinfection with Cnm-positive S. mutans and Campylobacter rectus resulted in a significant increase in urinary protein among IgAN patients ${ }^{28}$.

In conclusion, the present results suggest that $\mathrm{Cnm}$-positive $\mathrm{S}$. mutans organisms harbored in the oral cavity are associate with IgAN severity via their colonization in the tonsils. This may support the significance of tonsillectomy as treatment for IgAN patients and the importance of oral care for the prevention of kidney disease. We propose a new concept of 'oral-kidney association', considering that $S$. mutans may affect the pathogenesis of IgAN. In order to further investigate the concept that intraoral infection can cause and aggravate IgAN, accumulation of additional clinical evidence is needed.

\section{Materials and Methods}

Subjects and clinical data. The subjects $(n=61)$ were treated for IgAN as outpatients at the National Defense Medical College Hospital, Tokorozawa, Japan and Seirei Hamamatsu General Hospital, Hamamatsu, Japan. Each had been previously diagnosed with IgAN based on kidney biopsy results, with histological diagnosis made based on light microscopy and IF findings. All patients were over 15 years of age and underwent tonsillectomy between 2006 and 2017 at the National Defense Medical College Hospital or Seirei Hamamatsu General Hospital $^{27}$. For patients aged 15-19 years, the consent for our study protocol was obtained not only from them but also from their parents. Exclusion criteria were diagnosis of a secondary IgAN diseases (e.g. IgA vasculitis, lupus nephritis) and treatment with steroid or immunosuppression therapy. The control group $(\mathrm{n}=40)$ was consisted of patients requiring tonsillectomy for chronic tonsillitis. At the time of the tonsillectomy, urinary protein excretion, urinary occult blood, urinary protein excretion/urinary creatinine excretion, urinary RBC count, serum creatinine, eGFR, serum IgA, serum albumin, serum total cholesterol, serum low-density lipoprotein cholesterol, serum triglyceride, blood pressure (systolic, diastolic), height, body weight, and body mass index were obtained. $\mathrm{RBC}$ score was defined as urinary $\mathrm{RBC}<5 /$ high power field (HPF): 0; 5-10/HPF: $1 ; 10-30 / \mathrm{HPF}: 2$; and $>30$ / $\mathrm{HPF}: 3$.

Generation of antiserum against $\mathrm{Cnm}$. The bacterial cells used in this analysis were from Cnm-positive and Cnm-negative strains. The Cnm-positive strains were SA31, TW295, and TW871 [referred to as Cnm (+) S. mutans]. Those were isolated from separate individual but were the same, as they each expressed Cnm protein. Cnm-negative strains included the standard strain MT8148 [referred to as Cnm (-) S. mutans] and its Cnm-defective isogenic mutant strains, including TW295CND [referred to as Cnm- knockout (KO) S. mutans], MSSA Terashima, and E. coli coliB.

We constructed TW295CND (Cnm-KO S. mutans) to be used as a negative control during western blotting and immunostaining with the $\mathrm{Cnm}$ antiserum. This mutant strain with insertional inactivation of the $\mathrm{cnm}$ gene was constructed as previously reported ${ }^{41}$. First, the $\mathrm{cnm}$ gene was amplified by PCR using TaKaRa Ex Taq (Takara Bio. Inc., Kusatsu) with primers constructed based on the $\mathrm{cnm}$ sequence from strain TW295 (GenBank accession no. AB469913). The amplified fragment was subsequently cloned into a pGEM-T Easy vector (Promega, Madison, WI) to generate pTN11. The middle of the open reading frame of $\mathrm{cnm}$ in pTN11 was cleaved by BsmI and was then ligated with the erythromycin resistance gene $(\mathrm{erm})$ from the recombinant plasmid, which was linearized by digestion at the unique restriction site of PstI. Next, the plasmid was introduced into $S$. mutans TW295 using a homologous recombination method. The transformants were screened on MS agar plates 
(A)

Cnm

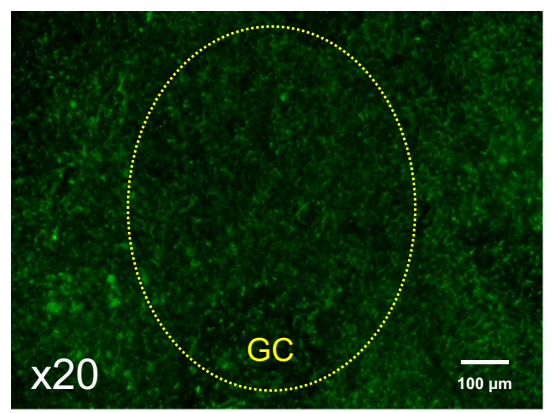

(B)

CD68

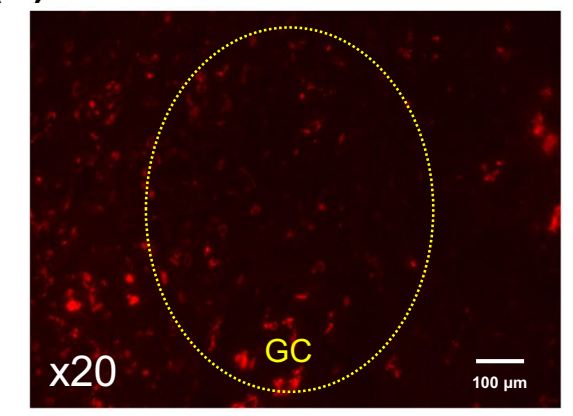

(C) Nuclear

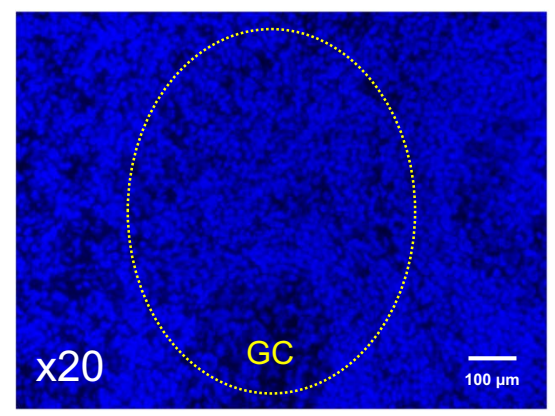

(D) Merged

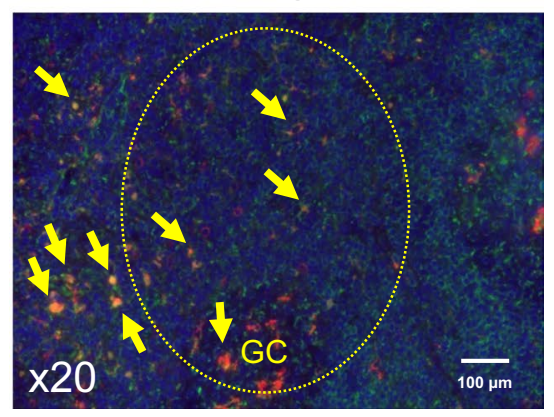

Figure 4. Double IF staining for Cnm and CD68 on tonsillar sections from IgAN patients. (A-D) Double IF staining for Cnm (Alexa Fluor 488: green) and CD68 (Alexa Fluor 594: red) and counterstaining of nuclei (DAPI: blue) were performed using tonsillar sections previously stained with immunoperoxidase for Cnm. (A) $\mathrm{Cnm}$ was predominantly observed in the germinal centers (GC) or surrounding area, with the surrounding area being more dominant. (B) CD68, a macrophage marker, was observed in the germinal centers and surrounding area. (C) Nuclear staining with DAPI. (D) Merged image of (A-C). Arrows indicate Cnm-positive macrophages (original magnification, A-D $\times 20$ ). Findings suggest that $\mathrm{Cnm}$ coexist with macrophages in the tonsils.

containing erythromycin $(10 \mathrm{mg} / \mathrm{mL})$. The $\mathrm{Cnm}$ antiserum was generated by intramuscular administration of $\mathrm{rCnm}$ in rabbits, as previously described ${ }^{42,43}$. Briefly, antiserum against $\mathrm{Cnm}$ was generated by injecting rabbits (New Zealand white rabbits; Oriental Yeast Co. Ltd., Tokyo) with 4 intramuscular injections of purified rCnm 
$(1200 \mu \mathrm{g})$ emulsified with a block copolymer adjuvant (Titer-Max Gold; CytRx Co., Atlanta, GA) every 2 weeks. The antibody titer of each antiserum sample was confirmed by enzyme-linked immunosorbent assay (ELISA) using $\mathrm{rCnm}$. We collected blood from the rabbits prior to immunizing (day 0) and after the third injection of the $\mathrm{rCnm}$ /adjuvant (day 35). We then confirmed that the antibody titers were adequately elevated using ELISA. Specifically, after dilution of serums by 6400-25600 times, the absorbance ratios (day35/0) at an OD of $450 \mathrm{~nm}$ ranged from 22.6-28.3. To verify the specificity of the Cnm antiserum, western blotting of rCnm and whole bacterial cell extracts was performed.

Light microscopic immunohistochemistry was performed on smears with Cnm (+) S. mutans, Cnm (-) S. mutans, and Cnm-KO S. mutans using Cnm antiserum, with counterstaining performed with Mayer's hematoxylin (Wako Chemicals, Osaka). As for the preparation of culture smear, we applied $5 \mu \mathrm{L}$ of bacteria cultured in brain-heart Infusion medium $(10 \mathrm{~mL})$ to a slide glass with a sterile pipette and left it to stand for approximately 30 minutes to dry. Next, we dipped and fixed the slide glass in methanol for 2 minutes and then left the slide glass for approximately 30 minutes to dry.

The Ethics Committee of Animal Care and Experimentation, National Defense Medical College, Japan, approved all requests for animals and the intended procedures of the present study (permission no. 19044). All experiments were performed in accordance with relevant guidelines and regulations.

Checking Cnm antiserum specificity. Fixing of tonsillar and kidney samples was performed with $10 \%$ formalin, and they were subsequently embedded in paraffin. For specificity verification, Cnm antiserum saturated with $\mathrm{rCnm}$ protein was used for immunoperoxidase staining of serial tonsillar sections.

Assessment of $\mathrm{Cnm}$ positivity in tonsils of IgAN patients and chronic tonsillitis. For serial tonsillar sections, immunoreactivity against $\mathrm{Cnm}$ was quantitatively evaluated following two-step indirect immunoperoxidase staining for $\mathrm{Cnm}$. Four images of each specimen were randomly obtained at low magnification and were subsequently analyzed using ImageJ-Fiji programs (ImageJ2, National Institutes of Health image) ${ }^{44,45}$ to determine the ratio of the area with Cnm positivity in the entire tonsil.

$S$. mutans strains were isolated from the saliva and confirmed using a previously described method ${ }^{40}$. Briefly, salivary specimens were collected in sterile phosphate-buffered saline and streaked onto mitis salivarius agar plates containing bacitracin and sucrose. We chose five colonies from each specimen and then extracted the genomic DNA from each strain. To confirm the identity of $S$. mutans, PCR was performed using $S$. mutans-specific primers sets (forward, 5'-GGC ACC ACA ACA TTG GGA AGC TCA GTT-3'; reverse, 50-GGA ATG GCC GCT AAG TCA ACA GGA T-3'), as previously described ${ }^{46}$. PCR was also performed to detect $c n m$-positive strains using $\mathrm{cnm}$-specific primers sets (forward, 5'-GAC AAA GAA ATG AAA GAT GT-30; reverse, 50-GCA AAG ACT CTT GTC CCT GC- $3^{\prime}$ ), as previously described ${ }^{22}$.

Histological studies. The Oxford classification of IgAN includes four pathological variables that independently determine the risk for developing progressive renal disease-namely, mesangial hypercellularity (M0/ M1 lesion), segmental glomerulosclerosis (S0/S1 lesion), endocapillary hypercellularity (E0/E1 lesion), and tubular atrophy/interstitial fibrosis (T0/T1/T2 lesion $)^{47,48}$. We compared the renal histology of Cnm-positive and Cnm-negative groups of IgAN patients using the Oxford classification in a blind test.

IF staining on tonsillar and kidney sections. We performed IF staining of renal biopsy specimens and tonsillar specimens obtained from patients who underwent tonsillectomy after having been diagnosed with IgAN and in whom both kidney and tonsillar specimens were available. Indirect double IF staining with Cnm antiserum and a mouse monoclonal antibody against human CD68, a marker for macrophages (PG-M1, Agilent), was performed using the following secondary antibodies: Alexa Fluor 488-labeled donkey anti-rabbit immunoglobulin G (IgG; Molecular Probes, Eugene, OR) and Alexa Fluor 594-labeled donkey anti-mouse IgG (Molecular Probes).

IF staining for Gd-IgA1 in kidney tissues from IgAN patients was performed using KM55, a Gd-IgA1- specific monoclonal antibody that has specific immunoreactivity for glomerular IgA deposition and can thus reflect the pathogenesis of $\operatorname{IgAN}^{49}$. Gd-IgA1 staining has been reported to be useful for diagnosis of $\operatorname{IgAN}^{50}$ and is hence performed with biopsy samples at many institutions, including ours, for such diagnosis. The Alexa Fluor 488-labeled donkey anti-rabbit IgG (Molecular Probes) secondary antibody was used for detection. Based on the degree of staining, IgAN patients were divided into two groups: (i) strong $(1+)$ and (ii) weak $( \pm)$ (there was no patient of negative $(-)$ staining). We calculated the percentage of the number of patients in (i) to the number of ones in the IgAN group.

Ethics. This study was conducted in full adherence to the Declaration of Helsinki (64th WMA General Assembly, Fortaleza, Brazil, 2013), and the study protocol was approved by the Ethics Committee of National Defense Medical College (approval no. 2690) and Seirei Hamamatsu General Hospital (approval no. 1807). All subjects were informed about the protocol and provided written consent prior to their participation.

Statistics. All quantitative values are expressed as means \pm standard deviation. When a significant difference was found, further statistical analysis of the difference between groups was performed using Fisher's PLSD test, Mann-Whitney U test, or Fisher's exact test, with $p<0.05$ considered to indicate statistical significance. All statistical analyses were performed using the JMP software package (version 14; SAS Institute Inc., Cary, NC).

Received: 20 February 2019; Accepted: 17 December 2019;

Published online: 27 December 2019 


\section{References}

1. Emancipator, S. N. \& Lamm, M. E. IgA nephropathy: pathogenesis of the most common form of glomerulonephritis. Lab. Invest. 60, 168-183 (1989).

2. Levy, M. \& Berger, J. Worldwide perspective of IgA nephropathy. Am. J. Kidney Dis. 12, 340-347 (1988).

3. Manno, C. et al. A novel simpler histological classification for renal survival in IgA nephropathy: a retrospective study. Am. J. Kidney Dis. 49, 763-775 (2007).

4. D'Amico, G. The commonest glomerulonephritis in the world: IgA nephropathy. Q. J. Med. 64, 709-727 (1987).

5. Julian, B. A., Waldo, F. B., Rifai, A. \& Mestecky, J. IgA nephropathy, the most common glomerulonephritis worldwide. A neglected disease in the United States? Am. J. Med. 84, 129-132 (1988).

6. Wyatt, R. J. \& Julian, B. A. IgA nephropathy. N. Engl. J. Med. 368, 2402-2414 (2013).

7. Donadio, J. V. \& Grande, J. P. IgA nephropathy. N. Engl. J. Med. 347, 738-748 (2002).

8. Hotta, O. et al. Tonsillectomy and steroid pulse therapy significantly impact on clinical remission in patients with IgA nephropathy. Am. J. Kidney Dis. 38, 736-743 (2001).

9. Hirano, K. et al. Association Between Tonsillectomy and Outcomes in Patients With Immunoglobulin A Nephropathy. JAMA network open 2, e194772 (2019).

10. Komatsu, H. et al. Effect of tonsillectomy plus steroid pulse therapy on clinical remission of IgA nephropathy: a controlled study. Clin. J. Am. Soc. Nephrol. 3, 1301-1307 (2008).

11. Kennoki, T., Ishida, H., Yamaguchi, Y. \& Tanabe, K. Proteinuria-reducing effects of tonsillectomy alone in IgA nephropathy recurring after kidney transplantation. Transplantation 88, 935-941 (2009).

12. Ushigome, H. et al. Efficacy of tonsillectomy for patients with recurrence of IgA nephropathy after kidney transplantation. Clin. Transplant. 23(Suppl 20), 17-22 (2009).

13. Watanabe, H. et al. Comprehensive microbiome analysis of tonsillar crypts in IgA nephropathy. Nephrol. Dial. Transplant. 32, 2072-2079 (2017).

14. Suzuki, S., Nakatomi, Y., Sato, H., Tsukada, H. \& Arakawa, M. Haemophilus parainfluenzae antigen and antibody in renal biopsy samples and serum of patients with IgA nephropathy. Lancet 343, 12-16 (1994).

15. Kusano, K., Tokunaga, O., Ando, T. \& Inokuchi, A. Helicobacter pylori in the palatine tonsils of patients with IgA nephropathy compared with those of patients with recurrent pharyngotonsillitis. Hum. Pathol. 38, 1788-1797 (2007).

16. Koyama, A. et al. Staphylococcus aureus cell envelope antigen is a new candidate for the induction of IgA nephropathy. Kidney Int. 66, 121-132 (2004).

17. Rollino, C., Vischini, G. \& Coppo, R. IgA nephropathy and infections. J. Nephrol 29, 463-468 (2016).

18. Nagasawa, Y. et al. Periodontal disease bacteria specific to tonsil in IgA nephropathy patients predicts the remission by the treatment. PLoS One 9, e81636 (2014).

19. Kojima, A. et al. Infection of specific strains of Streptococcus mutans, oral bacteria, confers a risk of ulcerative colitis. Sci. Rep. 2, 332 (2012).

20. Nakano, K. \& Ooshima, T. Serotype classification of Streptococcus mutans and its detection outside the oral cavity. Future Microbiol. 4, 891-902 (2009).

21. Nomura, R. et al. Potential high virulence for infective endocarditis in Streptococcus mutans strains with collagen-binding proteins but lacking PA expression. Arch. Oral Biol. 58, 1627-1634 (2013).

22. Nakano, K. et al. The collagen-binding protein of Streptococcus mutans is involved in haemorrhagic stroke. Nat. Commun. 2, 485 (2011).

23. Tonomura, S. et al. Intracerebral hemorrhage and deep microbleeds associated with cnm-positive Streptococcus mutans; a hospital cohort study. Sci. Rep. 6, 20074 (2016).

24. Watanabe, I. et al. Oral Cnm-positive Streptococcus Mutans Expressing Collagen Binding Activity is a Risk Factor for Cerebral Microbleeds and Cognitive Impairment. Sci. Rep. 6, 38561 (2016).

25. Naka, S. et al. A specific Streptococcus mutans strain aggravates non-alcoholic fatty liver disease. Oral Dis. 20, 700-706 (2014).

26. Misaki, T. et al. Distribution of Streptococcus mutans strains with collagen-binding proteins in the oral cavity of IgA nephropathy patients. Clin. Exp. Nephrol. 19, 844-850 (2015).

27. Misaki, T. et al. Presence of Streptococcus mutans strains harbouring the $\mathrm{cnm}$ gene correlates with dental caries status and IgA nephropathy conditions. Sci. Rep. 6, 36455 (2016).

28. Misaki, T. et al. Campylobacter rectus in the Oral Cavity Correlates with Proteinuria in Immunoglobulin A Nephropathy Patients. Nephron 139, 143-149 (2018).

29. Kim, J. K. et al. Clinical features and outcomes of IgA nephropathy with nephrotic syndrome. Clin. J. Am. Soc. Nephrol. 7, 427-436 (2012).

30. Dizin, E. et al. Albuminuria induces a proinflammatory and profibrotic response in cortical collecting ducts via the $24 \mathrm{p} 3$ receptor. Am. J. Physiol. Renal Physiol. 305, F1053-1063 (2013).

31. Hiki, Y. et al. Mass spectrometry proves under-O-glycosylation of glomerular IgA1 in IgA nephropathy. Kidney Int. 59, 1077-1085 (2001).

32. Odani, H. et al. Direct evidence for decreased sialylation and galactosylation of human serum IgA1 Fc O-glycosylated hinge peptides in IgA nephropathy by mass spectrometry. Biochem. Biophys. Res. Commun. 271, 268-274 (2000).

33. Caraman, P. L. et al. Mucosal infections and allergy in IgA nephropathy. A retrospective study. Association des Nephrologues de l'Est de la France. Contrib. Nephrol. 104, 24-30 (1993).

34. Bene MC, F. G., Hurault de Ligny, B., Kessler, M. \& Duheille, J. Immunoglobulin A nephropathy. Quantitative immunohistomorphometry of the tonsillar plasma cells evidences an inversion of the immunoglobulin A versus immunoglobulin G secreting cell balance. J. Clin. Invest. 71, 6 (1983).

35. Nakata, J. et al. Changes in nephritogenic serum galactose-deficient IgA1 in IgA nephropathy following tonsillectomy and steroid therapy. PLoS One 9, e89707 (2014).

36. Hoefakker, S. et al. Immunohistochemical detection of co-localizing cytokine and antibody producing cells in the extrafollicular area of human palatine tonsils. Clin. Exp. Immunol. 93, 223-228 (1993).

37. Horie, A. et al. IgA1 molecules produced by tonsillar lymphocytes are under-O-glycosylated in IgA nephropathy. Am. J. Kidney Dis. 42, 486-496 (2003).

38. Boyd, J. K. \& Barratt, J. Immune complex formation in IgA nephropathy: CD89 a 'saint' or a 'sinner'? Kidney Int. 78, 1211-1213 (2010).

39. de Tymowski, C. et al. CD89 Is a Potent Innate Receptor for Bacteria and Mediates Host Protection from Sepsis. Cell reports 27, 762-775.e765 (2019).

40. Nakano, K. et al. Development of a PCR method for rapid identification of new Streptococcus mutans serotype k strains. J. Clin. Microbiol. 42, 4925-4930 (2004).

41. Nakano, K. et al. Molecular characterization of Streptococcus mutans strains containing the cnm gene encoding a collagen-binding adhesin. Arch. Oral Biol. 55, 34-39 (2010).

42. Matsumoto-Nakano, M., Fujita, K. \& Ooshima, T. Comparison of glucan-binding proteins in cariogenicity of Streptococcus mutans. Oral Microbiol. Immunol. 22, 30-35 (2007). 
43. Nomura, R. et al. Identification and characterization of a collagen-binding protein, Cbm, in Streptococcus mutans. Mol. Oral Microbiol. 27, 308-323 (2012).

44. Rueden, C. T. et al. ImageJ2: ImageJ for the next generation of scientific image data. BMC Bioinformatics 18, 529 (2017).

45. Schindelin, J. et al. Fiji: an open-source platform for biological-image analysis. Nature methods 9 , 676-682 (2012).

46. Hoshino, T. et al. PCR detection and identification of oral streptococci in saliva samples using gtf genes. Diagn. Microbiol. Infect. Dis. 48, 195-199 (2004).

47. Roberts, I. S. et al. The Oxford classification of IgA nephropathy: pathology definitions, correlations, and reproducibility. Kidney Int. 76, 546-556 (2009).

48. Cattran, D. C. et al. The Oxford classification of IgA nephropathy: rationale, clinicopathological correlations, and classification. Kidney Int. 76, 534-545 (2009).

49. Suzuki, H. et al. IgA nephropathy and IgA vasculitis with nephritis have a shared feature involving galactose-deficient IgA1-oriented pathogenesis. Kidney Int. 93, 700-705 (2018).

50. Wada, Y. et al. Clinical significance of serum and mesangial galactose-deficient IgA1 in patients with IgA nephropathy. PLoS One 13, e0206865 (2018).

\section{Acknowledgements}

This work was partially supported by JSPS KAKENHI (Nos JP19K10098). We thank Prof. Masashi Tsunoda (Department of Preventive Medicine and Public Health, National Defense Medical College) for the kind support as well as our colleague Keiko Komoda for the expert secretarial assistance.

\section{Author contributions}

S.I., T.M., Y.N., M.M.N. and K.N. designed and performed the experiments under the supervision of H.K. and N.O. Antiserum against Cnm was generated by R.N., O.M., S.N. and K.W., and clinical specimens processing was done by S.I. and T.M. Result were analyzed by S.I., T.M., R.N., S.N. and K.W. The manuscript was written by S.I., T.M., Y.N., R.N. and K.N., with the final version approved by all of the authors.

\section{Competing interests}

The authors declare no competing interests.

\section{Additional information}

Correspondence and requests for materials should be addressed to S.I.

Reprints and permissions information is available at www.nature.com/reprints.

Publisher's note Springer Nature remains neutral with regard to jurisdictional claims in published maps and institutional affiliations.

(c) (i) Open Access This article is licensed under a Creative Commons Attribution 4.0 International License, which permits use, sharing, adaptation, distribution and reproduction in any medium or format, as long as you give appropriate credit to the original author(s) and the source, provide a link to the Creative Commons license, and indicate if changes were made. The images or other third party material in this article are included in the article's Creative Commons license, unless indicated otherwise in a credit line to the material. If material is not included in the article's Creative Commons license and your intended use is not permitted by statutory regulation or exceeds the permitted use, you will need to obtain permission directly from the copyright holder. To view a copy of this license, visit http://creativecommons.org/licenses/by/4.0/.

(C) The Author(s) 2019 